\title{
Electrophoretic Mobility of a Cylinder with High Surface Charge Density
}

\author{
W. P. J. T. VAN DER DRIFT, A. DE KEIZER, AND J. TH. G. OVERBEEK \\ Van 't Hoff Laboratory, University of Utrecht, Padualaan 8, Utrecht, The Netherlands
}

Received January 15, 1979; accepted March 19, 1979

\begin{abstract}
The electrophoretic mobility of a cylindrical particle is calculated by combining the solution of the complete Poisson-Boltzmann equation in cylindrical coordinates with the equations of Henry. Results are obtained for pointlike counterions (Gouy-Chapman picture) and for counterions having a finite diameter (Stern layer model). The deformation of the ionic atmosphere under the influence of the external field (relaxation effect) is not taken into account. In application to actual data a correction procedure for the relaxation effect, such as the semiempirical method proposed by W. J. H. M. Möller, G. A. J. van Os, and J. Th. G. Overbeek (Trans. Faraday Soc. 57, 312, 325, 1961), should be combined with the present treatment. Results can be applied to stiff (e.g., DNA) and to flexible (e.g., vinylic polyacids) polyelectrolytes.
\end{abstract}

\section{INTRODUCTION}

Natural or synthetic dissolved linear polyelectrolytes such as nucleic acids, polyphosphates, and vinylic polyacids can be considered to behave approximately as a series of randomly oriented cylinders.

A complete theory of electrophoretic mobility for cylinders is lacking, but would be highly desirable, because electrophoresis is a relatively simple technique for investigating the electric and hydrodynamic properties of charged particles. An early attempt is due to Henry (1). Gorin (see Abramson et al. (2)) has worked out Henry's results for a limited number of cases of low $\zeta$-potential. We have shown previously (3) how solutions for cylinders oriented perpendicularly or parallel to the applied field should be combined to give results for arbitrary orientation.

In this paper we extend Henry's and Gorin's treatments to high surface potential by using the complete Poisson-Boltzmann equation (in the following abbreviated as $\mathrm{P}-\mathrm{B}$ equation), still neglecting the relaxation effect. Moreover, we consider the influence of a charge-free layer between the surface charge and the countercharge in the solution (Stern model) and our approach is applicable to various locations of the slipping plane and to binding of counterions. In the Appendix we show that under many circumstances a flexible polyelectrolyte coil is so open that electric and hydrodynamic interactions between distant parts of the same chain and between parts of different chains are small and that therefore the cylinder is a much better model than the homogeneously charged sphere. This implies that the electrophoretic mobility (to be abbreviated as E.M.) of flexible polyions is nearly independent of their molecular weights and concentrations, and this is more accurately true, the higher the concentration of the supporting electrolyte.

If our results are used in combination with the semiempirical correction for the relaxation effect, as given by Möller et al. (4) (see also van der Drift and Overbeek (5)) a complete interpretation of the electrophoretic mobility can be obtained.

Recently Stigter (6) succeeded in a theoretical calculation of the relaxation effect for transversely oriented cylinders, but uncertainty still exists about the relaxation 
contribution for orientation parallel to the field, and thus the applicability of Stigters computations is still in doubt.

\section{BASIC IDEAS}

\subsection{Henry's Electrophoresis Equations:}

The Influence of Orientation

In his theory of the electrophoresis of infinitely long circular cylinders, Henry (1) has solved the problem for two extreme orientations. For cylinders with their axes parallel to the applied electric field, $X$, the electrophoretic velocity, $U_{\|}$, is given by

$$
\frac{U_{\|}}{X}=\frac{\epsilon \epsilon_{0} \psi_{a}}{\eta} .
$$

For cylinders perpendicular to the applied field, Henry's result for the electrophoretic mobility $\left(U_{\perp} / X\right)$ may be written after integration by parts as

$$
\begin{aligned}
\frac{U_{\perp}}{X}=\frac{\epsilon \epsilon_{0}}{2 \eta}\left\{\psi_{a}+2 a^{4} \int_{\infty}^{a} \frac{1}{r^{4}}\left(\frac{d \psi}{d r}\right) d r\right. \\
\left.-a^{2} \int_{\infty}^{a} \frac{1}{r^{2}}\left(\frac{d \psi}{d r}\right) d r\right\}
\end{aligned}
$$

or, after further integration by parts, as

$$
\begin{aligned}
\frac{U_{\perp}}{X}=\frac{\epsilon \epsilon_{0}}{\eta}\left\{\psi_{a}+4 a^{4} \int_{\infty}^{a} \frac{\psi}{r^{5}} d r\right. & \\
& \left.-a^{2} \int_{\infty}^{a} \frac{\psi}{r^{3}} d r\right\} .
\end{aligned}
$$

In these equations $\epsilon_{0}$ is the permittivity of the vacuum, $\epsilon$ is the dielectric constant of the medium, and $\eta$ its viscosity. $\psi$ is the potential in the medium at a distance $r$ from the cylinder axis, $a$ is the radius of the cylinder, and $\psi_{a}$ the potential at its surface, which is considered to be the slipping plane.

For low potentials, where the DebyeHückel linear approximation may be applied, $\psi(r)$ is the product of $\psi_{a}$ and a function of $\kappa r$, where

$$
\kappa^{2}=\frac{F^{2} \Sigma c_{i} z_{i}^{2}}{\epsilon \epsilon_{0} \mathbf{R} T}
$$

Here $F, \mathbf{R}$, and $T$ have their usual meaning, $c_{i}$ and $z_{i}$ are the concentration and charge number, respectively, of the ions of type $i$ in the bulk of the solution.

Then Eq. [2] or [3] may be replaced by

$$
\frac{U_{\perp}}{X}=\frac{\epsilon \epsilon_{0} \psi_{a}}{\eta F(\kappa a)} .
$$

De Keizer et al. (3) have shown that the average electrophoretic mobility $U / X$ of a randomly oriented cylinder is obtained from

$$
\frac{U}{X}=\frac{1}{3} \frac{U_{\|}}{X}+\frac{2}{3} \frac{U_{\perp}}{X},
$$

or defining

$$
\begin{aligned}
& \frac{1}{B}=\frac{1}{3}+\frac{2}{3 F(\kappa a)}, \\
& \frac{U}{X}=\frac{\epsilon \epsilon_{0} \psi_{a}}{\eta B} .
\end{aligned}
$$

Stigter (7) has extended the proof given by de Keizer et al. and he has shown that Eq. [6] is correct even if the relaxation effect is taken into account.

For Eq. [6] to be valid the applied field has to be so weak that it causes no appreciable orientations, the test being that the mobilities and conductivities are independent of the field strength.

The limits for $B$ and $F(\kappa a)$ are

$$
\begin{array}{ll}
\text { for } \kappa a \gg 1 & F(\kappa a)=1 \text { and } B=1, \\
\text { for } \kappa a \ll 1 & F(\kappa a)=2 \text { and } B=1.5 .
\end{array}
$$

The function $F(\kappa a)$ has been evaluated for $\kappa a$ values from 0.4 up to 3 by Gorin (see (2)), who applied the solution of the linearized $\mathbf{P}-\mathrm{B}$ equation for cylindrical symmetry. For higher surface charge density, however, the complete $\mathrm{P}-\mathrm{B}$ equation has to be used and $F\left(\kappa \alpha, \psi_{a}\right)$ depends not only on $\kappa a$ but also on the surface potential $\psi_{a}$.

\subsection{Stern Layer and Location of the Slipping Plane}

It is often necessary to take into account that a layer of solvent adheres firmly to the 
particle and that consequently the slipping plane is located at a certain distance from the interface between particle and dispersion medium. In that case the quantity, $a$, in Eq. [2] and the following has to be replaced by the hydrodynamic radius, $h$, of the particle plus adhering liquid. The potential at the slipping plane is usually written $\zeta\left(=\psi_{h}\right)$. Furthermore the usual form of the $\mathrm{P}-\mathrm{B}$ equation neglects the finite size of the ions. A simple correction for this defect, as proposed by Stern (8), is the assumption of a charge-free layer between the surface charge on the particle and the beginning of the diffuse double layer. It is frequently assumed that the slipping plane and the beginning of the diffuse double layer (Stern plane or outer Helmholtz plane, see Grahame (9)) are coincident. Another obvious choice for the slipping plane is the plane between the surface of the particle plus the adhering liquid and the first layer of counterions. Finally it may occur that the particle charge is modified by binding of counterions within the adhering solvent layer.

\section{DOUBLE LAYER AROUND A CYLINDRICAL PARTICLE}

\subsection{Approximate Analytical Solution of the Complete Poisson-Boltzmann Equation}

We have used the approximate analytical solution of the $\mathrm{P}-\mathrm{B}$ equation, as obtained by Philip and Wooding (10). These results can be substituted more easily in Henry's equations [1]-[3] than the corresponding numerical solutions of the $\mathrm{P}-\mathrm{B}$ equation. Furthermore, we extended the Philip and Wooding approximation to unsymmetrical electrolytes, and we have applied the Stern correction to account for the distance of closest approach of the counterions to the interface.

Consider an infinitely long, positively charged cylinder with radius, $a$, a smeared out surface charge density, $\sigma_{a}$, and surrounded by a $z_{+}-z_{-}$electrolyte with concentrations $c_{+}$and $c_{-}$(amounts of cations and anions per unit volume, respectively). The radius of the counterions is $a_{-}$. The radius $b\left(=a+a_{-}\right)$fixes the boundary of the charge-free Stern layer. Reversal of signs makes the results applicable to a negatively charged cylinder. The potential distribution in the static case (no external electrical field) is governed by the following equations:

$a \leqslant r \leqslant b$, Laplace equation:

$$
\frac{1}{r} \frac{d}{d r}\left(r \frac{d \psi}{d r}\right)=0
$$

with, at

$$
\begin{aligned}
& r=a, \quad \frac{d \psi}{d r}=-\frac{\sigma_{a}}{\epsilon \epsilon_{0}}, \\
& r=b, \quad \psi=\psi_{b} .
\end{aligned}
$$

$r \geqslant b$, Poisson-Boltzmann equation:

$$
\begin{array}{r}
\frac{1}{r} \frac{d}{d r}\left(r \frac{d \psi}{d r}\right) \\
=-\frac{F}{\epsilon \epsilon_{0}}\left(z_{+} c_{+} \exp \left(-\frac{z_{+} F \psi}{\mathbf{R} T}\right)\right. \\
\left.-z_{-} c_{-} \exp \left(\frac{z_{-} F \psi}{\mathbf{R} T}\right)\right),
\end{array}
$$

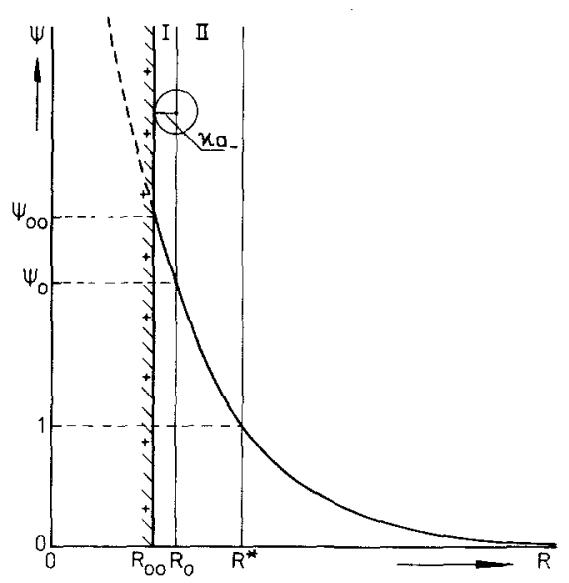

FIg. 1. The potential in the double layer around a charged cylindrical particle in dimensionless variables. I, Stern layer (Laplace equation). II, Inner region (only one exponential for the counterions). III, from $R^{*}$ to $R \rightarrow \infty$. Outer region (Debye-Hückel approximation). 
with, at

$r=b, \frac{d \psi}{d r}=\left(\frac{d \psi}{d r}\right)_{b}=-\frac{\sigma_{a}}{\epsilon \epsilon_{0}} \cdot \frac{a}{b}$,

$r=\infty, \quad \psi \rightarrow 0$.

According to Philip and Wooding the diffuse double layer is separated into two regions, an inner region with $r<r^{*}$ and an outer region with $r \geqq r^{*}$ (Fig. 1), where $r^{*}$ is de- fined by $r^{*}=r>b$ with $z_{-} F \psi\left(r^{*}\right) / \mathbf{R} T=1$. For the inner region the term with the negative exponential (co-ions) is neglected, while for the outer region the solution of the linearized $\mathrm{P}-\mathrm{B}$ equation is used. At the transition $\left(r=r^{*}\right)$ both the potential, $\psi$, and its first derivative $d \psi / d r$ are continuous.

The solutions can be advantageously presented in terms of the following dimensionless variables:

$$
\begin{aligned}
Z & \equiv\left(\frac{2 z_{-}}{z_{+}+z_{-}}\right)^{1 / 2}, \quad \text { for symmetrical electrolytes } Z=1, \\
R & \equiv \kappa r, \quad R_{00} \equiv \kappa a, \quad R_{0} \equiv \kappa b, \quad R^{*} \equiv \kappa r^{*}, \\
\Psi & \equiv \frac{z_{-} F \psi}{\mathbf{R} T}, \quad \Psi_{00} \equiv \frac{z_{-} F \psi_{a}}{\mathbf{R} T}, \quad \Psi_{0} \equiv \frac{z_{-} F \psi_{b}}{\mathbf{R} T}, \\
Y_{0} & \equiv \frac{a z_{-} F \sigma_{a}}{\epsilon \epsilon_{0} \mathbf{R} T} .
\end{aligned}
$$

Substituting the physical constants for water at $25^{\circ} \mathrm{C}(\epsilon=78.304)$ in Eqs. [4] and [14], we have

and

$$
\kappa=3.291\left(I \text { moles }^{-1} \text { liter }\right)^{1 / 2} \mathrm{~nm}^{-1}
$$

$$
Y_{0}=1.4315 z_{-} Q \mathrm{~nm},
$$

where $I\left(\equiv\left(c_{+} z_{+}^{2}+c_{-} z_{-}^{2}\right) / 2\right)$ is the ionic strength of the solution and $Q$ is the number of elementary charges per unit length of the cylinder. Although the electrophoretic mobility is more nearly proportional to the potential at the slipping plane (see Eq. [8]) than to the linear charge density, we express the mobility in terms of $Q$, since the charge of polyions can often be determined independently.

The equations to be solved are now:

$$
\begin{aligned}
R_{00} \leqslant R \leqslant R_{0}: & \frac{1}{R} \frac{d}{d R}\left(R \frac{d \Psi}{d R}\right)=0, \\
R_{0} \leqslant R \leqslant R^{*}: & \frac{1}{R} \frac{d}{d R}\left(R \frac{d \Psi}{d R}\right)=\frac{Z^{2} \exp \Psi}{2}, \\
R \geqslant R^{*}: & \frac{1}{R} \frac{d}{d R}\left(R \frac{d \Psi}{d R}\right)=\Psi .
\end{aligned}
$$

The boundary conditions are

$$
\begin{aligned}
R=R_{00}, & \frac{d \Psi}{d R}=-\frac{Y_{0}}{R_{00}}, \\
R=R_{0}, & \Psi=\Psi_{0},
\end{aligned}
$$




$$
\begin{aligned}
\frac{d \Psi}{d R} & =-\frac{Y_{0}}{R_{0}}, \\
R=R^{*}, \quad & =1, \\
\frac{d \Psi}{d R} & =-\frac{K_{1}\left(R^{*}\right)}{K_{0}\left(R^{*}\right)}, \\
R \rightarrow \infty, \quad \Psi & =0 .
\end{aligned}
$$

$K_{1}\left(R^{*}\right)$ and $K_{0}\left(R^{*}\right)$ are modified Bessel functions of $R^{*}$. If $\Psi_{0}<1$, Eq. [17] is used over the whole range of $R>R_{0}$, and Eqs. [16], [21], and [22] can be left out of consideration.

The complete system of equations [15][17] and boundary conditions [18]-[23] can be solved in analytical form. The solutions are

$R_{00} \leqslant R \leqslant R, \quad \Psi=\Psi_{0}+Y_{0} \ln \frac{R_{0}}{R}$,

$\frac{d \Psi}{d R}=-\frac{Y_{0}}{R}$

$R_{0} \leqslant R \leqslant R^{*}$, Table I of Philip and Wooding (10) gives five sets of equations for $\Psi$ and for $d \Psi / d R$ depending on the value of the parameter

$$
\begin{gathered}
C(\mathrm{symm})=\{2 \\
-\left[R^{*} K_{1}\left(R^{*}\right) /\right. \\
\left.\left.K_{0}\left(R^{*}\right)\right]\right\}^{2}-\mathrm{e} R^{* 2} .
\end{gathered}
$$

For unsymmetrical electrolytes all $R$ and $R^{*}$ in Philip and Wooding's table have to be replaced by $Z R$ and $Z R^{*}$, respectively, but $C$ becomes

$$
\begin{aligned}
& C(\text { unsymm })=\{2 \\
& -\left[R^{*} K_{1}\left(R^{*}\right) \digamma\right. \\
& \left.\left.K_{0}\left(R^{*}\right)\right]\right\}^{2}-\mathrm{e} Z^{2} R^{* 2}
\end{aligned}
$$

$$
\begin{aligned}
& R \geqslant R^{*}, \quad \Psi=\frac{K_{0}(R)}{K_{0}\left(R^{*}\right)}, \\
& \frac{d \Psi}{d R}=-\frac{K_{1}(R)}{K_{0}\left(R^{*}\right)} .
\end{aligned}
$$

Philip and Wooding point out that in certain cases the solution for the inner region shows a singularity for $R=R_{\text {crit }}$ where $\Psi$ and $d \Psi / d R$ go to infinity. Obviously the solution can only be used when $R_{0}>R_{\text {crit }}$.

\subsection{Comparison with the Numerical Solution}

We have compared the results with the (exact) numerical solution using the RungeKutta procedure RK 2 (AP 252) developed by Zonneveld (11). An initial guess was made for $\Psi$ at large $R$ in the region of low potential (Eq. [28]). Then with the aforementioned procedure values were computed for $\Psi_{00}$ and $(d \Psi / d R)_{00}$. The exact value of the last quantity is known from the boundary condition [18]. The accuracy of the numerical solution is better than $0.01 \%$. The calculated percentage error in $\Psi_{00}$ for the approximate analytical solution of Philip and Wooding is shown in Fig. 2. For 1-1 and 2-2 electrolytes the error in $\Psi_{00}$ is always smaller than $3.5 \%$. The error for the nonsymmetrical $1-2$ and 2-1 electrolytes is sometimes larger, but never exceeds $6 \%$ at $\Psi_{00} \sim 1$.

The percentage error in the dimensionless electrophoretic mobility, $E$ (see following section), in the approximation of Henry, is mainly due to the error in $\Psi_{00}$ and for most vinylic and polysaccharidic polyelec- 


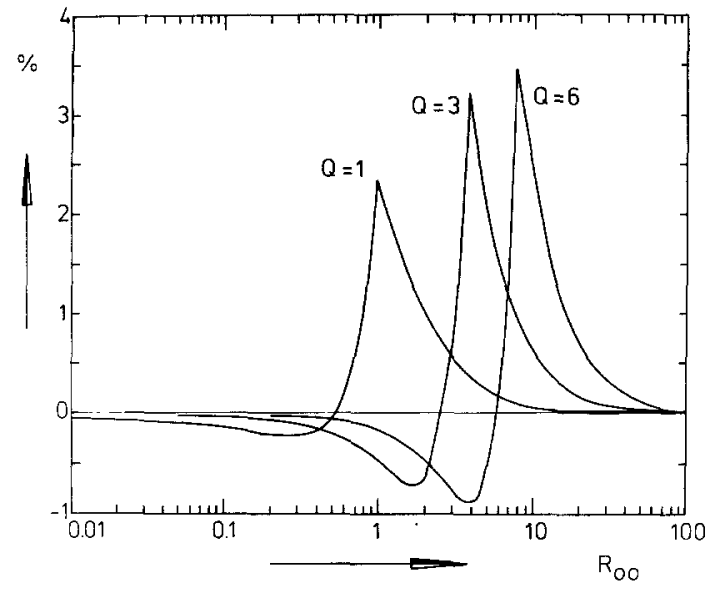

Fig. 2. Percentage error in $\Psi_{00}$ for 1-1 electrolytes as a function of $R_{00}=\kappa a$ at three different linear charge densities $Q=1,3$, and $6 \mathrm{~nm}^{-1}$. For vinylic polyelectrolytes $(a \sim 0.5 \mathrm{~nm})$ and double-stranded DNA $(a \sim 1 \mathrm{~nm}) R_{00}$ is smaller than 0.5 and 1 , respectively, provided the concentration of supporting 1-1 electrolyte does not exceed 0.1 mole/liter. A fully neutralized vinylic polyelectrolyte (e.g., polyacrylic acid) has a $Q$ of about $4 \mathrm{~nm}^{-1}$. The linear charge density of double-stranded DNA at $\mathrm{pH}=7$ amounts to about $6 \mathrm{~nm}^{-1}$. The sharp maximum in the curves occurs at $\Psi_{00}=1$.

trolytes and double-stranded DNA remains smaller than $0.5-1 \%$ if the electrolyte content does not greatly exceed $0.1 \mathrm{M}$. Consequently the approximate analytical solution of Philip and Wooding is acceptable for the calculation of the electrophoretic mobility of a large number of well-known macroions with a cylindrical electric double layer. For more details concerning the subject of this subsection see Van der Drift (12).

\section{CALCULATION OF THE ELECTROPHORETIC MOBILITY}

\subsection{Gouy-Chapman Approximation}

We define the dimensionless electrophoretic mobility, $E$, as

$$
E=\frac{2 \eta F z_{-}}{\epsilon \epsilon_{0} \mathbf{R} T} \cdot \frac{U}{X}, E_{\perp, \| l}=\frac{2 \eta F z_{-}}{\epsilon \epsilon_{0} \mathbf{R} T} \cdot \frac{U_{\perp, \|}}{X},
$$

and

$$
E=\frac{E_{\|}+2 E_{\perp}}{3}
$$

Henry's Eqs. [1] and [2] converted to the dimensionless form read

$$
\begin{aligned}
& E_{\|}=2 \Psi_{00}, \\
& E_{\perp}=\Psi_{00}+2 R_{00}^{4} \int_{\infty}^{R_{00}} \frac{d \Psi}{d R} \cdot \frac{1}{R^{4}} d R \\
& \quad-R_{00}^{2} \int_{\infty}^{R 00} \frac{1}{R^{2}} \frac{d \Psi}{d R} d R .
\end{aligned}
$$

By substituting Eqs. [24]-[29] into Eqs. [30]-[32] and taking $R_{00}=R_{0}$ we obtain solutions valid for pointlike ions and in the absence of a charge-free (Stern) layer. The calculations are performed with a Control Data Computer, Type Cyber 73-2 of the Academic Computer Centre, Utrecht, by the use of a suitable Algol-60 program. The surface potential $\Psi_{00}$, the actual potential $\psi_{a}$ in millivolts for water at $25^{\circ} \mathrm{C}$, and the dimensionless electrophoretic mobility, $E$, are calculated as functions of $z_{+}, z_{-}, R_{00}$ $(=\kappa a)$, and $Q$. For water at $25^{\circ} \mathrm{C}$ :

$$
\psi_{a}=\frac{\mathbf{R} T}{z_{-} F} \Psi_{00}=\frac{\Psi_{00}}{z_{-}} \times 25.693 \mathrm{mV}
$$

and

$\frac{U}{X}=\frac{\epsilon \epsilon_{0} \mathbf{R} T}{2 \eta z_{-} F}, \quad E=$

$$
\frac{E}{z_{-}} \times 1.00029 \times 10^{-4} \mathrm{~cm}^{2} \mathrm{~V}^{-1} \mathrm{sec}^{-1} .
$$

\subsection{Results}

The results of the calculations are presented in Figs. 3-6. Figure 3 shows how the electrophoretic mobility depends on $Q$ and on $\kappa a$. In Fig. 4, B (Eqs. [7] and [8]) is shown. It can be derived easily from Eqs. [8], [33], and [34] that $B=2 \Psi_{00} / E$. In the Debye-Hückel region $\left(\Psi_{00} \ll 1\right) B$ is only a function of $\kappa a$. There it can be described 


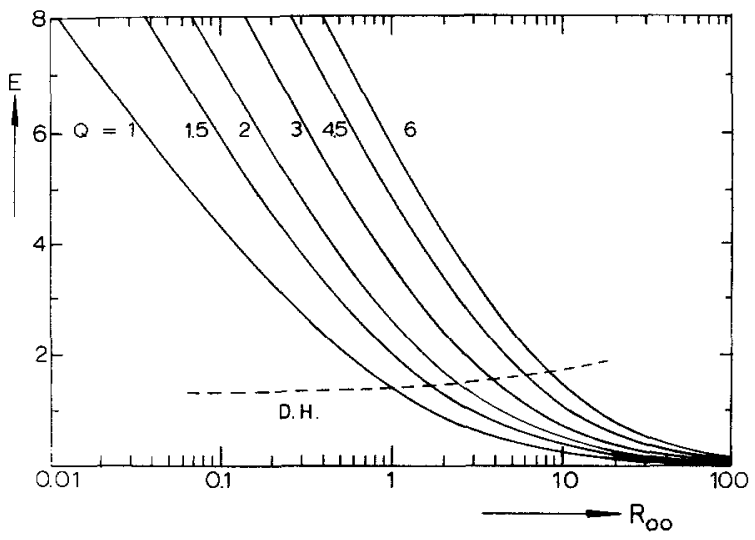

FIG. 3. Dimensionless electrophoretic mobility, $E$, plotted against $R_{00}=\kappa a$ at various linear charge densities $Q$ in elementary charges per nanometer. 1-1 electrolytes. Below the dashed line the DebyeHückel approximation applies.

with an accuracy of better than $0.1 \%$ by the equation:

$B(D-H)$

$$
=\frac{2.200+5.882 \kappa a+(\kappa a)^{2}}{1.4667+3.935 \kappa a+(\kappa a)^{2}} .
$$

Figure 4 shows that $Q$ has practically no influence on $B$ above $\kappa a=5$, since even for high values of $Q, \Psi_{00}$ remains below 1 . Even at lower values of $\kappa a$ the influence of $Q$ on $B$ remains modest, the maximum decrease of $B$ being $3 \%$ at $Q=6 \mathrm{~nm}^{-1}$ and $\kappa a \simeq 0.3$. This dependency of $B$ on $Q$ at high surface charge densities can be taken into account, in the interval $0.001 \leqslant \kappa a \leqslant 0.3$, with the following equation:

$$
\begin{aligned}
B= & B(D-H)-[Q\{0.0109+0.00386 \log (\kappa \alpha) \\
& \left.\left.+0.000533 \log ^{2}(\kappa a)\right\}-0.006\right] . \quad[36]
\end{aligned}
$$

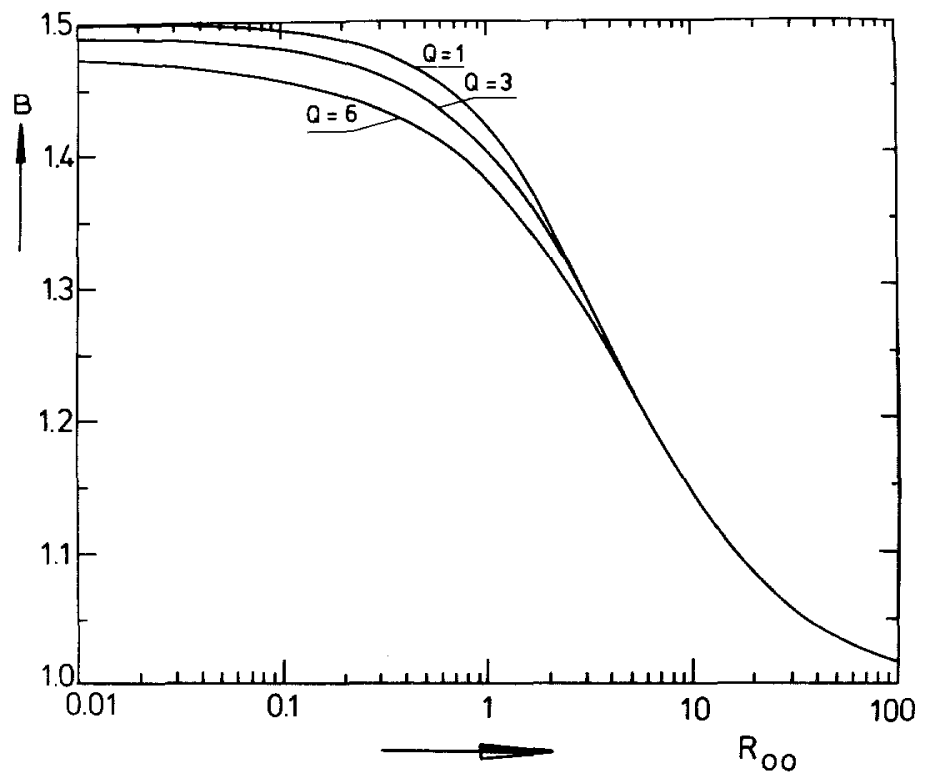

FIG. 4. Influence of $Q$ and $R_{00}$ on $B$ (Eq. [8]). The values for $Q \rightarrow 0$ coincide practically with those for $Q=1 \mathrm{~nm}^{-1}$. 


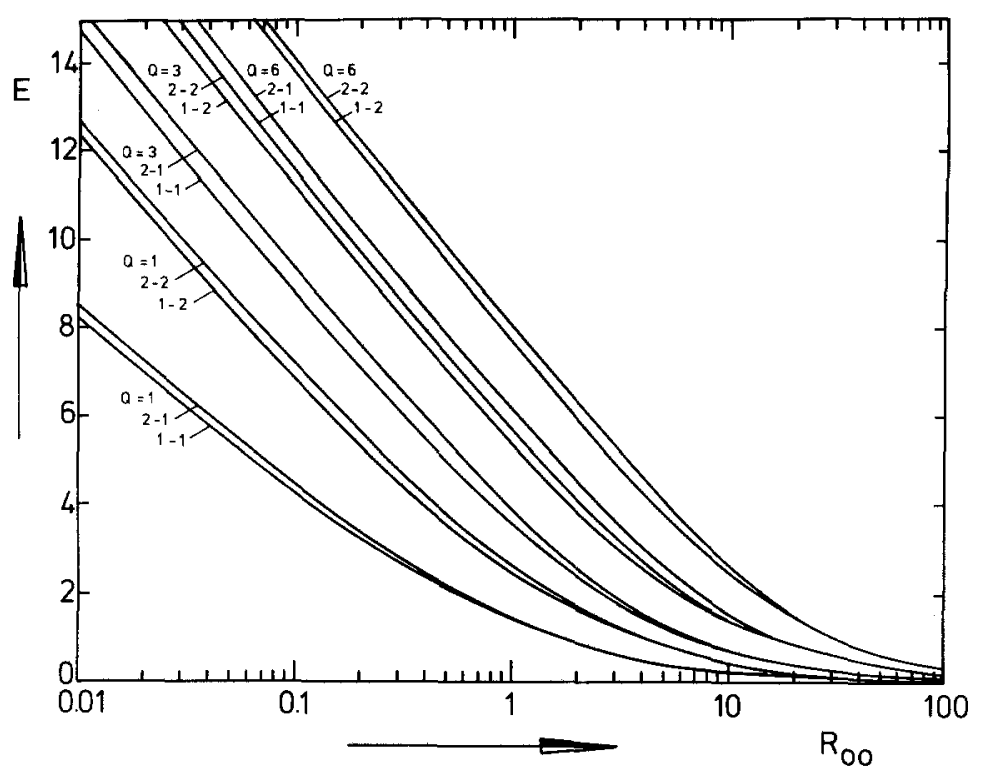

Fig. 5. Dimensionless electrophoretic mobility, $E$, for different $z_{+}-z_{-}$type electrolytes and for three values of $Q\left(1,3\right.$, and $\left.6 \mathrm{~nm}^{-1}\right)$.

The term within the square brackets is positive, if otherwise (e.g., for $Q=0$ ) its value is zero. The accuracy of Eq. [36] is within $0.1 \%$ for $0 \leqslant Q \leqslant 6 \mathrm{~nm}^{-1}$ except for $Q \simeq 6$ $\mathrm{nm}^{-1}$ and $\kappa a=0.3(+0.2 \%)$. For the range $0.3 \leqslant \kappa a \leqslant 6$,

$$
\begin{aligned}
B= & B(D-H) \\
- & {[Q\{0.00686-0.00599 \log (\kappa a)} \\
& \left.\left.-0.00258 \log ^{2}(\kappa a)\right\}-0.006\right] .
\end{aligned}
$$

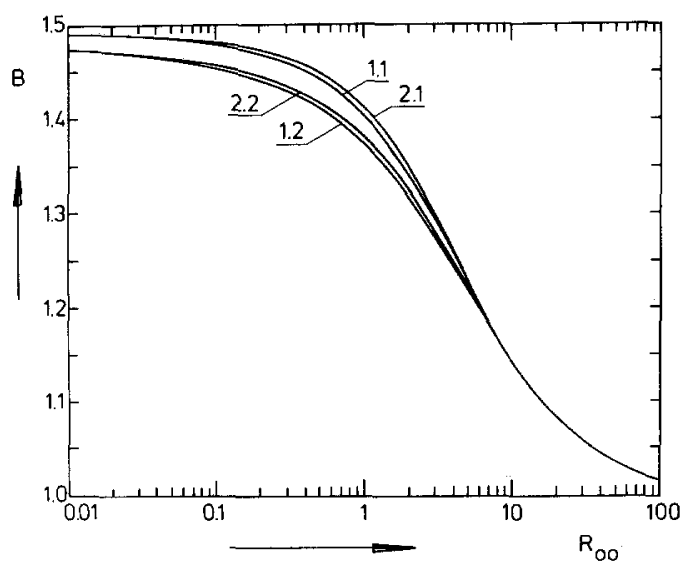

Fig. 6. Influence of electrolyte $\left(z_{+}-z_{-}\right)$on $B$ for $Q=3 \mathrm{~nm}^{-1}$.

Journal of Colloid and Interface Science, Vol. 71, No. 1, August 1979
The latter formula is valid for $0 \leqslant Q \leqslant 6$ $\mathrm{nm}^{-1}$ with an accuracy of $0.2 \%$. The term within the square brackets of Eq. [37] is zero if its value becomes negative.

Figures 5 and 6 show the influence of the charge numbers of counterions and co-ions on $E$ and on $B$. Bivalent counterions lower $B$ more strongly at the same $\kappa a$ than monovalent counterions. At high $\kappa a$ where the Debye-Hückel approximation applies $B$ is unaffected by the charge numbers of the ions.

\subsection{Influence of the Charge-Free Stern Layer and of Various Locations of the Slipping Plane}

The introduction of the Stern model in order to take the finite size of the counterions into account results in two effects. Calling the distance of closest approach of the center of the counterions to the cylinder surface (equal to the radius of the solvated counterion) $a_{-}$we obtain that the Gouy-Chapman layer extends from $\left(a+a_{-}\right)$to infinity rather than from $a$ to infinity. The potential at the Stern plane ( $R_{0}$ in Fig. 1 ) is then, for a given linear charge density $Q$, smaller than 
it would be if $a_{-}$were zero. At the cylinder surface $\left(R_{00}\right)$, however, the potential is larger than for $a_{-}=0$, because on the average the counterions are farther away from the surface. The exact course of the potential between $R_{00}$ and $R_{0}$ is described by the Laplace equation [24].

The effect of this modification in the ionic atmosphere on the E.M. depends on the situation of the slipping plane. If the slipping plane coincides with the Stern plane (an assumption often made in discussing electrophoresis, e.g., Lyklema (13)), the only modification that has to be made in the calculations of the previous subsection is the change of the cylinder radius $a$ into the larger hydrodynamic radius $\left(a+a_{-}\right)$ $=b$. This results in a lower E.M. for the same linear charge density, $Q$.

If the slipping plane remains located at the original cylinder surface, the Henry integrals (Eq. [32]) have to be extended through the charge-free layer with the use of Eq. [24] for the relation between $\Psi$ and $R$. This results in a larger E.M. at the same value of $Q$. Figure 7 illustrates the effect for a few realistic cases. It is clear that the change in mobility may become considerable when the ionic radius and the cylinder radius are of comparable magnitude.

Other locations of the slipping plane could be considered, e.g., between $R_{00}$ and $R_{0}$, for a cylinder with a solvation layer that is impenetrable for counterions of finite size or at $R>R_{0}$ (see following subsection), but the latter choice is then rather arbitrary. The calculations as such are easily made for any choice of the location of the slipping plane.

\subsection{Adsorption of Counterions}

Adsorbed counterions lower the effective charge of the cylinder. So do those ions in the ionic atmosphere that are situated inside the slipping plane. In both cases the E.M. will be lowered. It has to be assumed that all ions inside the slipping plane, whether adsorbed or not adsorbed, do not contribute

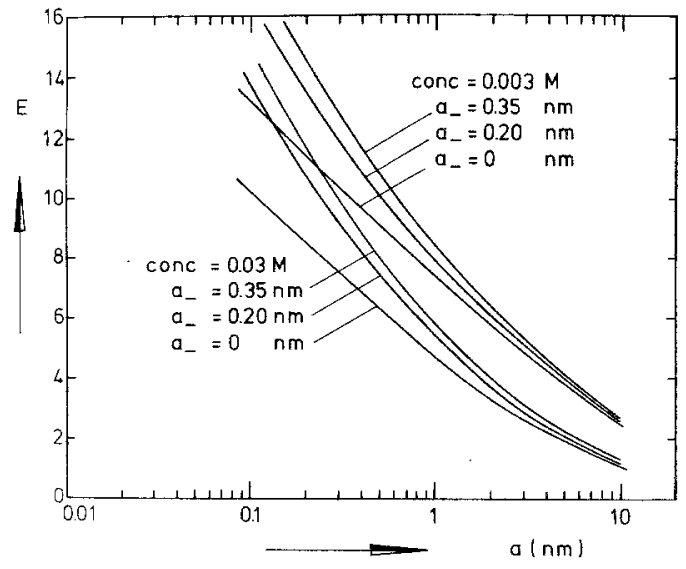

FIG. 7. Influence of the Stern layer on the electrophoretic mobility. The curves refer to the case in which the slipping plane is located at the original cylinder surface, $a$. The electrophoretic mobility for the case of the slipping plane coinciding with the Stern plane is found on the curves for $a_{-}=0$ but choosing the new radius $\left(a+a_{-}\right)=b$ instead of $a \cdot Q=3 \mathrm{~nm}^{-1}$, 1-1 electrolyte for all curves. Since absolute values ( 0.2 and $0.35 \mathrm{~nm}$, respectively) have been chosen for the thickness of the Stern layer, the abscissa in the figure is $a$ rather than $R_{00}$, and separate curves have to be given for different electrolyte concentrations.

any conductivity to the particle, since one of the basic assumptions made in deriving Henry's equations (Eqs. [2] and [3]) is that there is no conductance inside the slipping plane. For a given adsorption and a given location of the slipping plane the calculation of the mobility is straightforward using the techniques described above.

\section{COMPARISON WITH OTHER RECENT COMPUTATIONS}

A few recent papers have also dealt with the calculation of the electrophoresis of randomly oriented cylindrical particles. They are limited to 1-1 electrolytes and with the exception of Stigter's (6) paper also neglect the relaxation effect.

Nagasawa et al. (14) and Takahashi et al. (15) both obtain E.M.'s which are 20-30\% smaller than our values for the same parameters. The main part of the difference is due to their choosing the slipping plane to coin- 
cide with the Stern plane rather than with the surface of the cylinder. Furthermore, Nagasawa et al. approximate the Henry integrals by inserting $B=6 \pi \epsilon_{0}$ (in our notation with the rationalized four-quantity system, $B=1.5$ ) for all cases.

Mills (16), in solving the $P-B$ equation, introduced the method of piecewise linearization which results in slightly low values for $\Psi_{00}$ (a few percent). In the computation of the linear charge density of DNA from electrophoresis data obtained by Ross and Scruggs (17), Mills used Gorin's (2) values which are obtained by an incorrect averaging procedure (3) and are limited to the Debye-Hückel approximation. This results in an error of $-13.5 \%$ in the mobility at $Q=6 \mathrm{~nm}^{-1}$.

Stigter (6) did include the relaxation effect in his calculation of the electrophoresis of transversely oriented cylinders. He moreover concluded that for infinitely long cylinders parallel to the field, relaxation may be neglected and then he estimated the E.M. of randomly oriented cylinders and of open coils by using Eq. [6] of this paper. He found that the relaxation effect lowered the E.M. depending on $\Psi_{00}$ and on $\kappa a$ by 0 -about $20 \%$. In our opinion the relaxation effect of cylinders of finite length parallel to the field should not be neglected. It may even be rather large, due to the polarization of the double layer along the cylinder. Therefore we expect Stigter's estimate of the relaxation effect to be lower than the actual value.

\section{FINAL REMARK}

In our previous paper (5) we have shown that in certain cases empirical estimates of the relaxation effect on polyelectrolytes can be made. We shall combine the calculations of the E.M. without relaxation effect of the present paper with these estimates of the relaxation effect in the interpretation of measurements of the E.M. of polymethacrylate ions in a following paper (23).

\section{COMPUTER DATA}

A fairly extensive table of values of $\Psi_{00}$ and $E$ as functions of $Q$ and $\kappa a$ (38 values of $Q$ and 25 values of $\kappa a$ ) and the computer programs on which it is based are available on request from the authors. The computer programs are applicable to general $z_{+}-z_{-}$ electrolytes and include corrections for the Stern layer and for an arbitrary location of the slipping plane.

\section{APPENDIX}

Two rather different models have been proposed for describing the properties of coiled polyelectrolytes.

In the porous sphere model (18) the fixed charges on the polyelectrolyte are assumed to be homogeneously smeared throughout a sphere and the counterions and co-ions to be distributed inside and outside the sphere in accordance with the Poisson and the Boltzmann equations. The porous sphere is a good model, when the ionic atmospheres around the individual charges on the polyion overlap strongly, even when fairly distant parts of the polyion are considered.

On the other hand, when the thickness of the ionic atmosphere is small compared to the average distance between different chain elements the polyion is better represented by a loosely coiled cylinder surrounded by its ionic atmosphere.

The situation can be assessed in the following way. Debye and Bueche (19) have shown that a coiled polymer molecule with a radius of gyration $s$ may be represented as a homogeneous porous sphere with a radius $R_{\text {coil }}$ given by

$$
\left\langle R_{\text {coil }}^{2}\right\rangle=5 / 3\left\langle s^{2}\right\rangle \text {. }
$$

If the extended length of the coil is equal to $l$, we may consider the polymer or polyion as a cylinder surrounded by a cylindrical free space of radius $r_{\text {free }}, r_{\text {free }}$ being given by

$$
\pi r_{\text {free }}^{2} l=4 \pi R_{\mathrm{coil}}^{3} / 3
$$


The average distance between different parts of the chain is then of the order of $2 r_{\text {free }}$. If this distance is a good deal larger than the thickness of the ionic atmosphere, $1 / \kappa$, electric and hydrodynamic overlap are negligible and the cylinder model is to be preferred over the porous sphere model.

The actual data for most coiled polyions are such that $\kappa r_{\text {free }} \gg 1$. This is due to the fact that polyions are either stiff on account of their chemical structure (e.g., doublestranded DNA) or at least fairly stiff as a consequence of the mutual repulsion between adjacent charged groups.

We shall illustrate this more quantitatively with some computations on measured values of $R_{\text {coil }}$ (5) of half-neutralized PMA at different concentrations of supporting 1:1 electrolyte. $M_{\mathrm{PMA}}=270000 \mathrm{~g} \mathrm{~mole}^{-1}$. For the potential at $r_{\text {free }}$ the calculation is based on the method outlined in Section 3.1. For the velocity at $r_{\text {free }}$ we used Henry's equation (1) for the radius-dependent part of the liquid velocity, $v / X$, around a cylinder perpendicular to the applied field as given in Eq. [A3].

$$
\begin{aligned}
\frac{v\left(r=r_{\text {free }}\right)}{X} & =\frac{\epsilon \epsilon_{0}}{2 \eta} \int_{\infty}^{r_{\text {free }}}\left(\frac{d \psi}{d r}\right. \\
& \left.+a^{2} r \int_{\infty}^{r_{\text {free }}} \frac{1}{r^{3}} \nabla^{2} \psi d r\right) d r .
\end{aligned}
$$

Using the analogon of Eq. [30] we converted $v_{\text {free }}$ into a dimensionless velocity $E_{\text {free }}$ and compared this value to $E$ itself. The results are given in Table $I$.

It is obvious, that both the electric and hydrodynamic interaction between two parts of the coiled cylinder are very small indeed, especially at higher ionic strength. Consequently the cylinder model is to be preferred above the porous sphere model.

Two other important conclusions can be drawn at the same time. In the first place the E.M. of flexible polyelectrolytes is expected to be independent of its chain length, i.e., of its molecular weight $(20,21)$, even at low

\section{TABLE I}

Average Electric and Hydrodynamic Interaction between Distant Parts of a Coiled Cylinder ${ }^{a}$

\begin{tabular}{llllcl}
\hline $\begin{array}{c}c, 1-1 \\
\text { electrolyte } \\
\text { (mole liter }\end{array}$ & $\begin{array}{c}\left.R_{\text {coil }}\right) \\
(\mathrm{nm})\end{array}$ & $\begin{array}{c}r_{\text {free }} \\
(\mathrm{nm})\end{array}$ & $\kappa r_{\text {tree }}$ & $\Psi_{\text {fret }} / \Psi_{\text {o0 }}$ & $E_{\text {free }} / E$ \\
\hline 0.001 & 97 & 40.6 & 4.2 & $2.0 \times 10^{-3}$ & $2.3 \times 10^{-3}$ \\
0.003 & 87.5 & 34.7 & 6.3 & $2.5 \times 10^{-4}$ & $2.9 \times 10^{-4}$ \\
0.01 & 76.5 & 28.4 & 9.3 & $1.4 \times 10^{-5}$ & $1.7 \times 10^{-5}$ \\
0.03 & 63.5 & 21.5 & 12.2 & $8.8 \times 10^{-7}$ & $1.1 \times 10^{-6}$ \\
0.1 & 52 & 15.9 & 16.6 & $1.4 \times 10^{-8}$ & $1.8 \times 10^{-8}$ \\
\hline
\end{tabular}

${ }^{a}$ Data for half-neutralized polymethacrylic acid ( $M$ $=270,000 \mathrm{~g} \mathrm{~mole}-1$, contour length $=735 \mathrm{~nm}, Q$ $=2.13$ elementary charges per nanometer.

ionic strength (14), because in electrophoresis the coiled cylinder behaves as freely drained. Moreover, the same type of reasoning explains why the influence of the polyion concentration on its mobility is often negligible at low polyelectrolyte concentration and not too low ionic strength. As a matter of fact the concentration dependence of the mobility is due to hydrodynamic and electric overlap (22). We demonstrate this with the example of a solution of 0.01 monomole liter $^{-1}$ of polymethacrylate ions in a $0.01 \mathrm{M}$ solution of a 1-1 electrolyte, by considering the PMA as one giant chain. We define $r_{\text {free }}$ for this case as

$\pi r_{\text {free }}^{2} \times N_{\mathrm{Av}} \times l$

$$
\times 0.01 \text { mole liter }^{-1}=1,
$$

where $l=0.235 \mathrm{~nm}=$ the average length of a monomeric segment. Then we compare $r_{\text {free }}$ with the radius of the cylinder $(0.35 \mathrm{~nm})$ and the thickness of the double layer (3.16 $\mathrm{nm}$ ), and find

$$
\begin{aligned}
r_{\text {free }}= & 15.0 \mathrm{~nm} \\
& =(0.35+4.64 \times 3.16) \mathrm{nm} .
\end{aligned}
$$

At a distance of $4.64 \times(1 / \kappa)$ the electric potential is reduced to $<0.3 \%$ of its value at the cylinder surface, showing that the electric and thus the hydrodynamic overlap is negligible. 


\section{ACKNOWLEDGMENTS}

The authors are indebted to Miss Henny Miltenburg and Mrs. Marina Uit de Bulten for carefully typing the manuscript.

\section{REFERENCES}

1. Henry, D. C., Proc. Roy. Soc. Ser. A 133, 106 (1931).

2. Abramson, H. A., Moyer, L. S., and Gorin, M. H., "Electrophoresis of Proteins", p. 125. Reinhold, New York, 1942.

3. de Keizer, A., van der Drift, W. P. J. T., and Overbeek, J. Th. G., Biophys. Chem. 3, 107 (1975)

4. Möller, W. J. H. M., van Os, G. A. J., and Overbeek, J. Th. G., Trans. Faraday Soc. 57, 312, 325 (1961).

5. van der Drift, W. P. J. T., and Overbeek, J. Th. G., Rec. Trav. Chim. Pays-Bas 98, 81 (1979).

6. Stigter, D., J. Phys. Chem. 82, 1417 (1978).

7. Stigter, D., J. Phys. Chem. 82, 1424 (1978).

8. Stern, O., Z. Elektrochem. 30, 508 (1924).

9. Grahame, D. C., Chem. Rev. 41, 441 (1947).

10. Philip, J. R., and Wooding, R. A., J. Chem. Phys. 52, 593 (1970).

11. Zonneveld, J. A., “Automatic Numerical Integra- tion." Mathematisch Centrum, Amsterdam, 1964.

12. van der Drift, W. P. J. T., thesis, University of Utrecht. The Netherlands, 1975.

13. Lyklema, J., "Colloid and Interface Science" (M. Kerker, A. C. Zettlemoyer, and R. L. Rowell, Eds.), Vol. I, p. 257. Academic Press, New York, 1977.

14. Nagasawa, M., Noda, I., Takahashi, T., and Shimamoto, N., J. Phys. Chem. 76, 2286 (1972).

15. Takahashi, T., Noda, I., and Nagasawa, M., $J$. Phys. Chem. 74, 1280 (1970).

16. Mills, R. A., Biopolymers 9, 1511 (1970).

17. Ross, P. D., and Scruggs, R. L., Biopolymers 2, 231 (1964).

18. Hermans, J. J., and Overbeek, J. Th. G., Rec. Trav. Chim. Pays-Bas 67, 761 (1948); Wall, F. T., and Berkowitz, J., J. Chem. Phys. 26, 114 (1957); Lifson, S., J. Chem. Phys. 27, 700 (1957).

19. Debye, P., and Bueche, A. M., J. Phys. Chem. 16, 573 (1948).

20. Noda, I., Nagasawa, M., and Ota, M., J. Amer. Chem. Soc. 86, 5075 (1964).

21. Eisenberg, H., J. Polym. Sci. 30, 47 (1958).

22. Overbeek, J. Th. G., and Stigter, D., Rec. Trav. Chim. Pays-Bas 75, 543 (1956).

23. van der Drift, W. P. J. T., and Overbeek, J. Th. G. J. Colloid Interface Sci. 71, 79-92. 